\title{
Extraction optics for high lumen density sources
}

\author{
Dick K. G. de Boer ${ }^{*}$ (iD and Ludo Haenen
}

\begin{abstract}
For lighting applications demanding high brightness, a new type of LED-based light source has been developed, the high lumen density (HLD) source (luminance $>2 \cdot 10^{9} \mathrm{~cd} / \mathrm{m}^{2}$ ). The performance can be improved further, among other things by optimizing the concentrator used as extraction optics. For this optimization, much insight is obtained by ray-tracing simulations. The efficiency can be improved by more than $20 \%$ if a high-index concentrator could be used. Furthermore, it is possible to optimize the shape of the concentrator. For a low-index $(n \approx 1.5)$ concentrator, the light leakage can be reduced, but the gain in useful light is only marginal. For a high-index $(n \approx 1.8)$ concentrator, the gain in useful light amounts to a few percent.
\end{abstract}

Keywords: Collimation, Light-emitting diodes, Luminescence, Optical design, High-brightness source

\section{Introduction}

There are a number of lighting applications demanding high lumen density (high brightness), in which gas discharge lamps are not yet replaced by solid-state lighting solutions. Examples are digital projection, spot lighting (like entertainment lighting and architectural lighting), automotive front lighting, microscopy and endoscopy. In these applications, a high intensity is required in a small étendue (essentially surface area times solid angle). Laser-based sources are increasingly being used, but they have some disadvantages like being relatively expensive. Moreover, both discharge lamps and laser-based sources degrade relatively fast. Recently [1-5], we developed a new type of LED-based light source, the high lumen density (HLD) source, which is very stable and enables the mentioned applications (luminance $>2 \cdot 10^{9} \mathrm{~cd} / \mathrm{m}^{2}$ ). Similar concepts were investigated by others $[6,7]$.

The core of these light sources are converter rods, which consist of transparent (i.e., non-scattering) luminescent material that converts light from blue pump LEDs (Fig. 1). Whereas part of the converted light will escape from the long sides of the rod, the greater part is guided by total internal reflection (TIR) towards the small end facets. At one side, a mirror is placed to redirect all light to the other small facet, where it is extracted by a solid compound parabolic concentrator (CPC). In

\footnotetext{
*Correspondence: dick.de.boer@signify.com

Signify Research, High Tech Campus 7, 5656AE Eindhoven, The Netherlands
}

fact, the CPC has two functions: it extracts the light from the rod and also gives the light beam a welldefined exit area and angular divergence that suit the application.

The converter rod serves as a luminescent concentrator, of which the principle is well known from its use in luminescent solar concentrators [8-11]. In such a device, solar light is converted to light of longer wavelengths that is guided towards photovoltaic cells at the small sides of the concentrator plate. Whereas the étendue of an optical system cannot be decreased in the absence of conversion, it is possible to decrease étendue in a system with conversion. It can be shown [12] that the maximum concentration in terms of radiance is

$$
C_{\max }=\frac{n_{\text {out }}^{2} E_{\text {out }}^{3}}{n_{\mathrm{in}}^{2} E_{\mathrm{in}}^{3}} \exp \left(\frac{E_{\mathrm{in}}-E_{\text {out }}}{\mathrm{k} T}\right)
$$

where $n_{\text {in }}$ and $n_{\text {out }}$ are the refractive indices, respectively, of the media of the incoming and outgoing light and $E_{\text {in }}$ and $E_{\text {out }}$ are the energies of the incident and converted radiation, respectively. The thermodynamic background of this is that the entropy $\left(E_{\text {in }}-E_{\text {out }}\right) / T$, associated with the heat generated in the luminescence process, can be exploited to lower the entropy of the light. The underlying microscopic cause is that the luminescent sites behave as independent sources after conversion.

The conversion efficiency, defined as the ratio of the green flux emitted by the module and the blue flux 


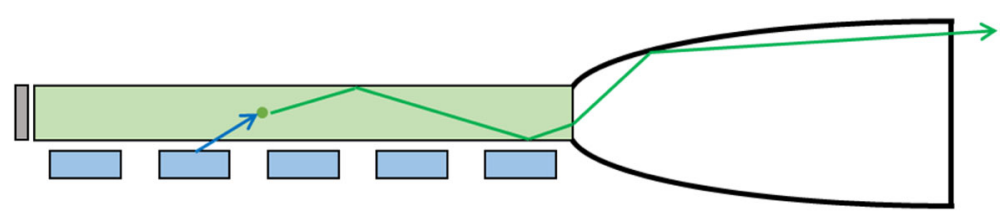

Fig. 1 Schematic drawing of high lumen density (HLD) module. The light from blue LEDs is converted to green light in the luminescent rod. Most of this light stays guided in the rod till it is extracted by the CPC at the right. At the left, a mirror is placed

emitted by the LEDs, is 0.28 for a typical HLD module [4], consisting of a block-shaped rod made of ceriumdoped lutetium aluminum garnet (LuAG:Ce, dimensions $64 \times 1.9 \times 1.2 \mathrm{~mm}^{3}$ ) with a glass CPC attached to it. Figure 2 shows the measured output spectrum from the module (red solid line), together with its absorption spectrum (blue) and its intrinsic emission spectrum (measured using a thin layer of powder, orange). The output spectrum is red shifted, since green light can be reabsorbed in the overlapping region with the absorption spectrum and subsequently reemitted.

The red dashed line shows the simulated output spectrum, obtained from ray-tracing simulations $[1,4]$ using the measured absorption (blue) and emission (orange) spectra as input. The simulated value for the conversion efficiency is 0.29 , slightly more than the experimental value.

The main reasons for this relatively low conversion efficiency were discussed before $[1,4]$ and will be repeated briefly here. Firstly, the injection of blue pump light is not fully efficient since part is reflected and part is transmitted. The conversion in the rod from blue to green light is inevitably associated with energy loss (Stokes shift). Also, the quantum efficiency (QE) is not unity. In the used materials, the measured QE is 0.95 or higher, whereas thermal quenching can be considered as negligible. Then, the converted light is emitted isotropically. Part of it will escape from the long sides of the rod and only light emitted at angles larger than the critical angle for TIR will stay guided in the rod. Additional guiding loss occurs after reabsorption and reemission of green light and because of scatter in the material. The measured scatter length is $400 \mathrm{~mm}$, resulting in only small loss in $64 \mathrm{~mm}$ length. Surface scatter can cause additional loss.

The converted light guided to the rod front end is extracted into the CPC. In the used module, not all light can be extracted because the CPC has a lower index than the rod. As will be discussed below, more light may be coupled out by using a high-index CPC [13]. Some additional loss may occur at the interface where the $\mathrm{CPC}$ is attached to the rod with glue.

Next, the light is collimated by reflection in the CPC and extracted in air from the front side. The collimation efficiency will not reach unity for a three-dimensional $\mathrm{CPC}$. In the remainder of this paper, we will discuss extensively this efficiency and how it can be improved.

\section{Methods}

Simulations were performed using LightTools raytracing software ([1], https://www.synopsys.com/opticalsolutions/lighttools.html). The LEDs are approximated by simple rectangular sources with a Lambertian distribution. The luminescent rod material has a wavelengthdependent refractive index and absorption coefficient (cf. Fig. 2). The emission spectrum is approximated by

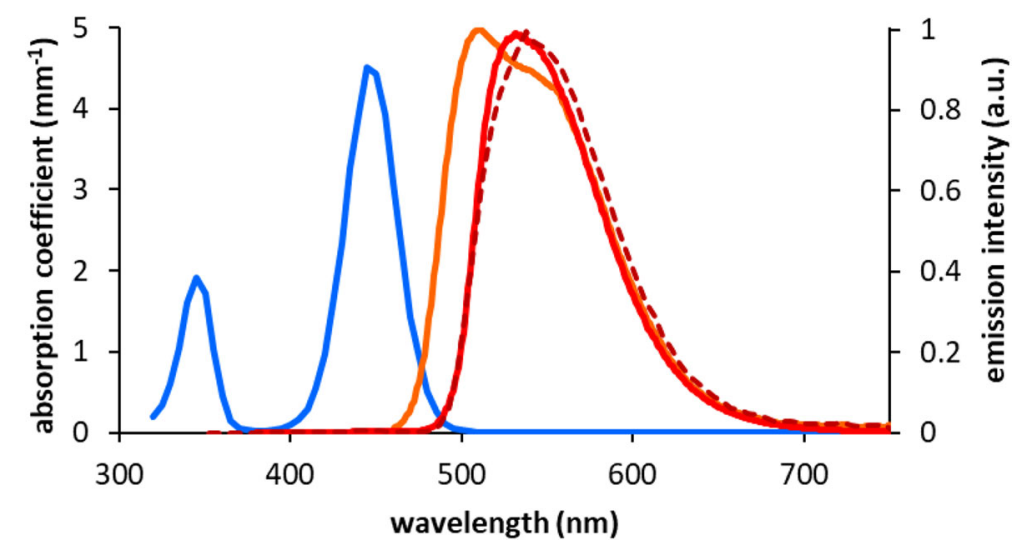

Fig. 2 Absorption and emission spectra of LuAG:Ce. Absorption (blue), emission of powder (orange) and from a CPC attached to a rod (red). The simulated emission spectrum is shown with red dashes 


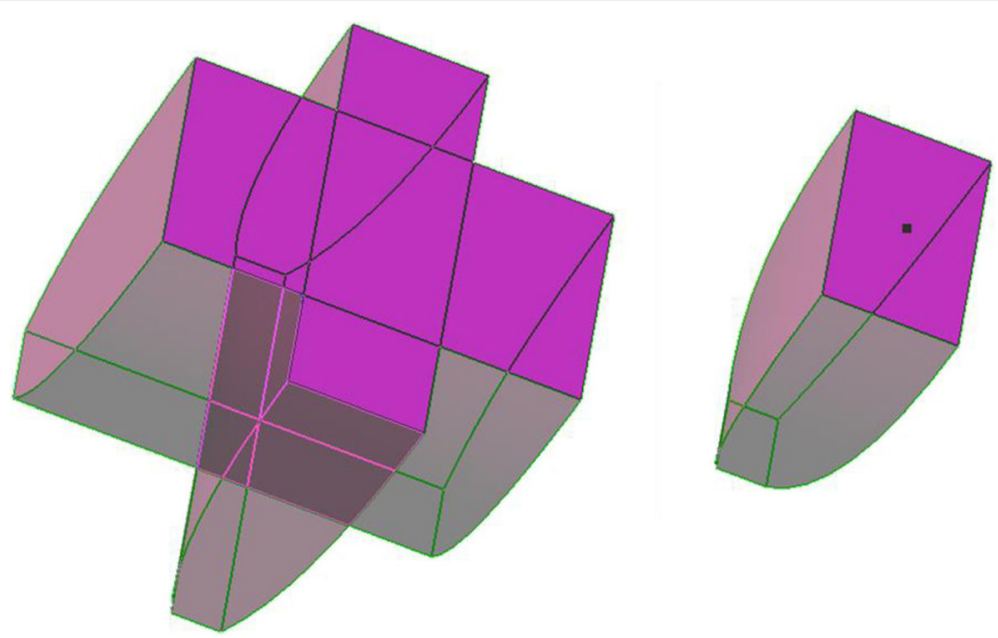

Fig. 3 Crossed CPC constructed by intersecting two extruded CPCS

that of a fine powder (cf. Fig. 2) and $\mathrm{QE}=0.95$. It is assumed that the volume scatter of the luminescent material is described by a Henyey-Greenstein model (https:// www.synopsys.com/optical-solutions/lighttools.html) with a scatter length of $400 \mathrm{~mm}$ and anisotropy factor 0.85 , whereas surface scatter is neglected. The rectangular CPCs are described as crossed CPCs [14]. That is, they are constructed by intersecting two extruded CPCs, see Fig. 3), of which the shortest one is stretched to ensure the same length as the longest one. The other parameters are given in the Results section. The optimized concentrators are described as rectangular 'Skin Solids' with 'Skin Surfaces' described as Bézier curves. Each Bézier curve is described by three control points, two of which are coincident with the endpoints of the curve [15]. Two parameters of the center control point can be varied (see Fig. 4). Receivers are placed at various positions in the system (see Figs. 5, 6, 7 and 8), where the number of hits at the receivers is used to assess the intensity distributions at different stages.

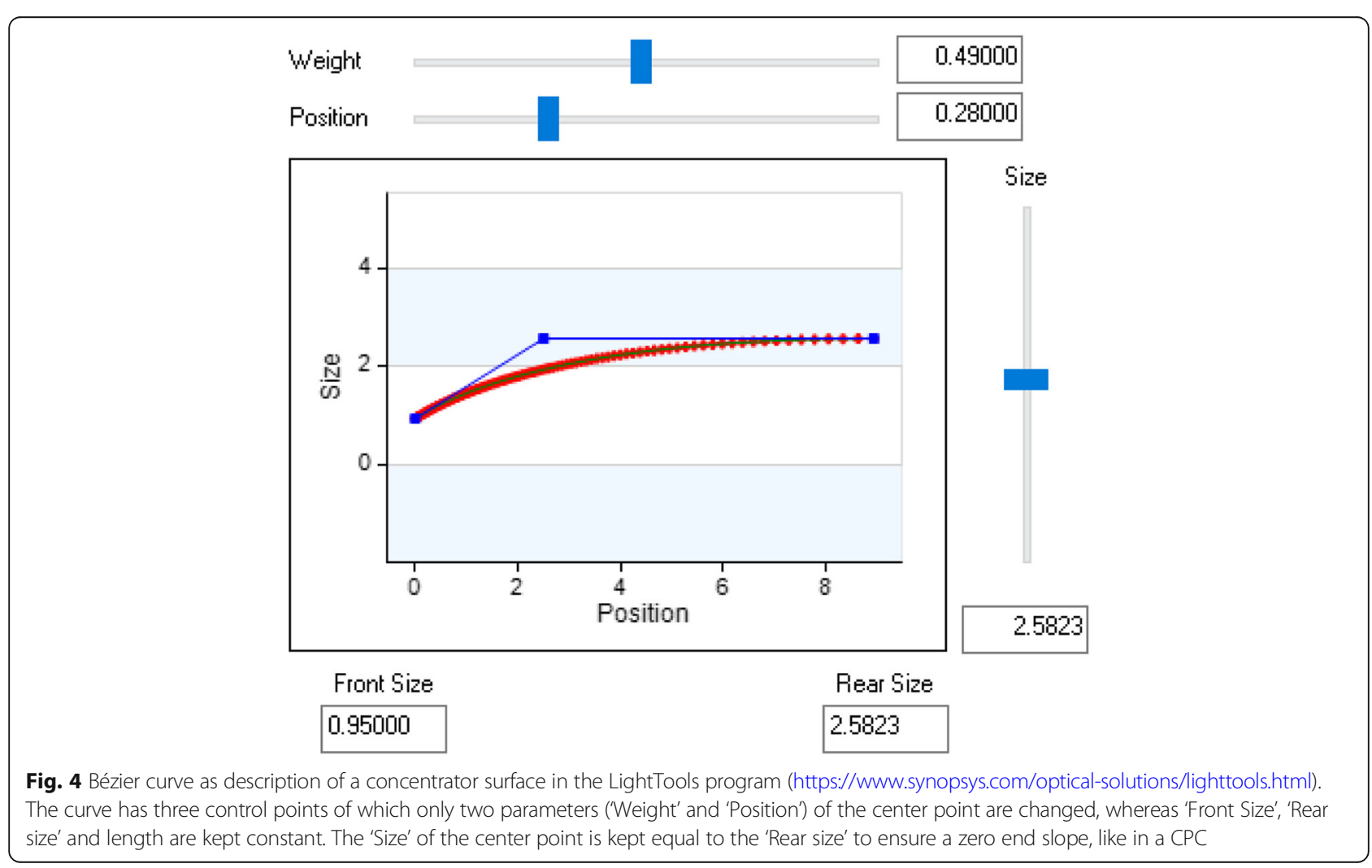




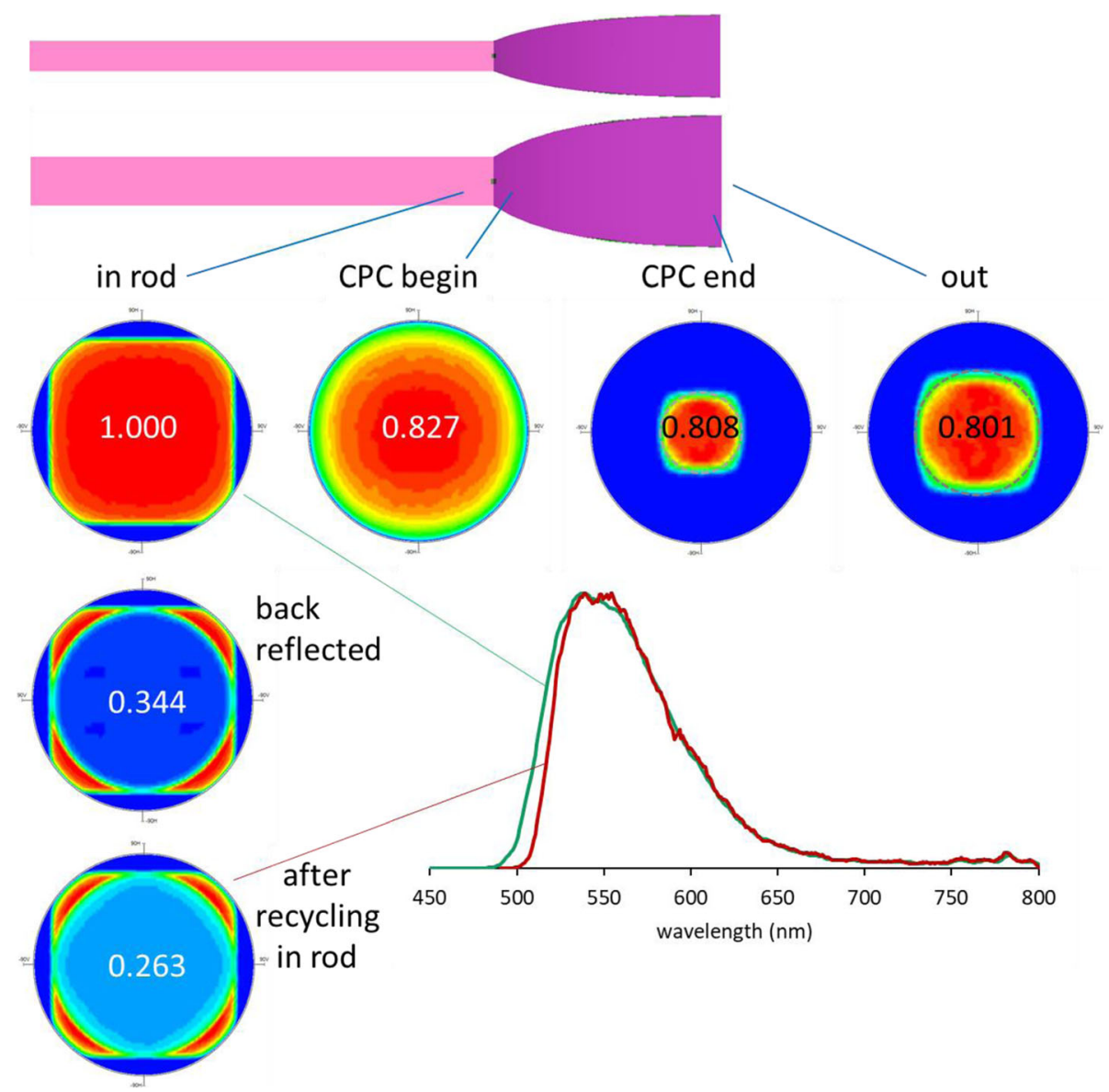

Fig. 5 Side views of a LuAG:Ce rod with a glass $(n=1.52)$ CPC attached to it with simulated angular and (normalized) spectral distributions of light at different stages. The numbers in the angular distributions show the fractions of light at the different stages

The mentioned conversion efficiencies have been determined in an integrating sphere (Instrument Systems) by separate measurements of the blue flux from the LED module and the green flux from the complete HLD module (at the same input electrical power). More details on the set-ups for the experimental results, as well as on the module construction, can be found in literature $[1-5]$.

\section{Results}

We first will consider a converter rod made of LuAG:Ce with a glass $\mathrm{CPC}$ attached to it. In the wavelength region of emission, LuAG has a refractive index $n=1.83$ and the used glass has $n=1.52$. The critical angle for TIR at the rod-air interface is $34^{\circ}$; the critical angle at the rodglass interface is $56^{\circ}$. In a two-dimensional picture, the light guided in the rod hits the rod-glass interface at angles of at most $\left(90^{\circ}-34^{\circ}=\right) 56^{\circ}$ and all light could be extracted in the glass CPC. In three dimensions, however, there are skew rays that are reflected, since they hit the rod-glass interface at higher angles.
To study this, we performed ray-tracing simulations on a LuAG:Ce rod with dimensions $64 \times 1.9 \times 1.2 \mathrm{~mm}^{3}$ with a rectangular concentrator attached to it. To suit the (digital projection) application, the chosen aspect ratio is $19: 12$, the exit étendue is $16.5 \mathrm{~mm}^{2}$ sr and the exit angle is $34^{\circ}$. The concentrator is designed to have this exit angle of $34^{\circ}$ in air and an entrance angle $\theta=90^{\circ}$ in glass. For an ideal concentrator, the étendue of $16.5 \mathrm{~mm}^{2}$ sr would be conserved and hence the étendue at the concentrator entrance, $n^{2} A \pi \sin ^{2} \theta$, is the same, with $n=1.52$, area $A=1.9 \times 1.2 \mathrm{~mm}^{2}$ and $\theta=90^{\circ}$.

First, we consider a concentrator consisting of two two-dimensional CPCs (Fig. 3). The cross section with entrance width $1.9 \mathrm{~mm}$ is just a normal two-dimensional $\mathrm{CPC}$, whereas the cross section with entrance width 1.2 $\mathrm{mm}$ (shown at the top of Fig. 5) is a stretched CPC. In Fig. 5, simulated angular light distributions (perpendicular to the plane of drawing) are shown at different stages in the system. The distribution shown top left is that inside the CPC, which extends up to $90^{\circ}$ at skew angles. The corresponding étendue amounts to $21 \mathrm{~mm}^{2} \mathrm{sr}$. Below it, the angular distribution of the radiation 

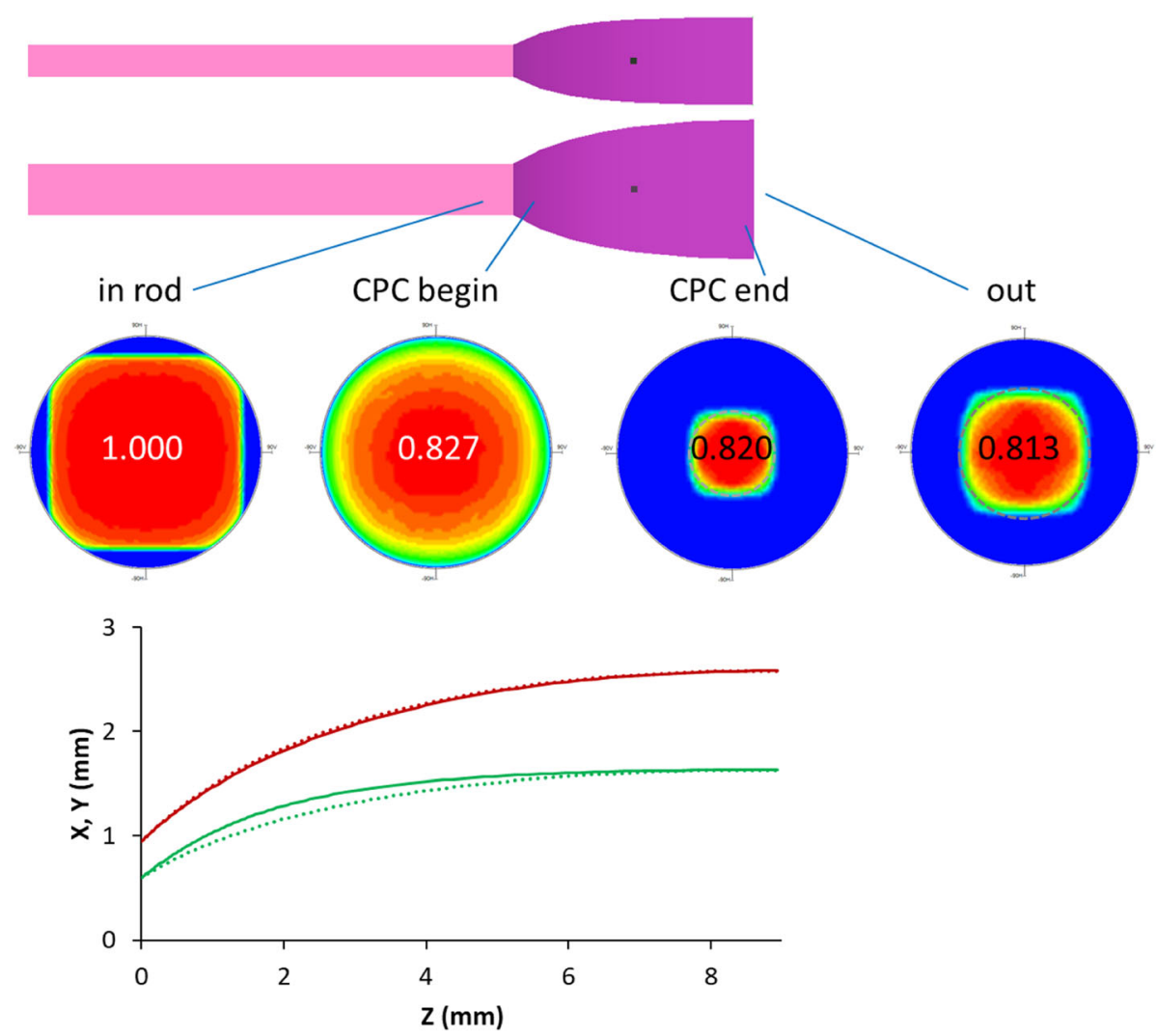

Fig. 6 Side views of a LuAG:Ce rod with a glass $(n=1.52)$ concentrator with optimized Bézier shape attached to it with simulated angular distributions of light at different stages. The numbers in the angular distributions show the fractions of light at the different stages. At the bottom the Bézier shapes of the two sides are shown (solid lines) together with the CPC shapes (dotted) of Fig. 5

reflected back from the rod-glass interface is shown, which corresponds to a fraction 0.344 of the light reaching the front of the rod. Rays in a cone with angles up to $56^{\circ}$ with the front surface have been transmitted, corresponding to a fraction of 0.656 . The total fraction of light in the CPC after the rod-glass interface, however, is 0.827 . There is additional light because the reflected light is recycled in the rod, as can be seen from the distribution shown bottom left. Note that, after TIR to the sides, light will return with the same angle and cannot

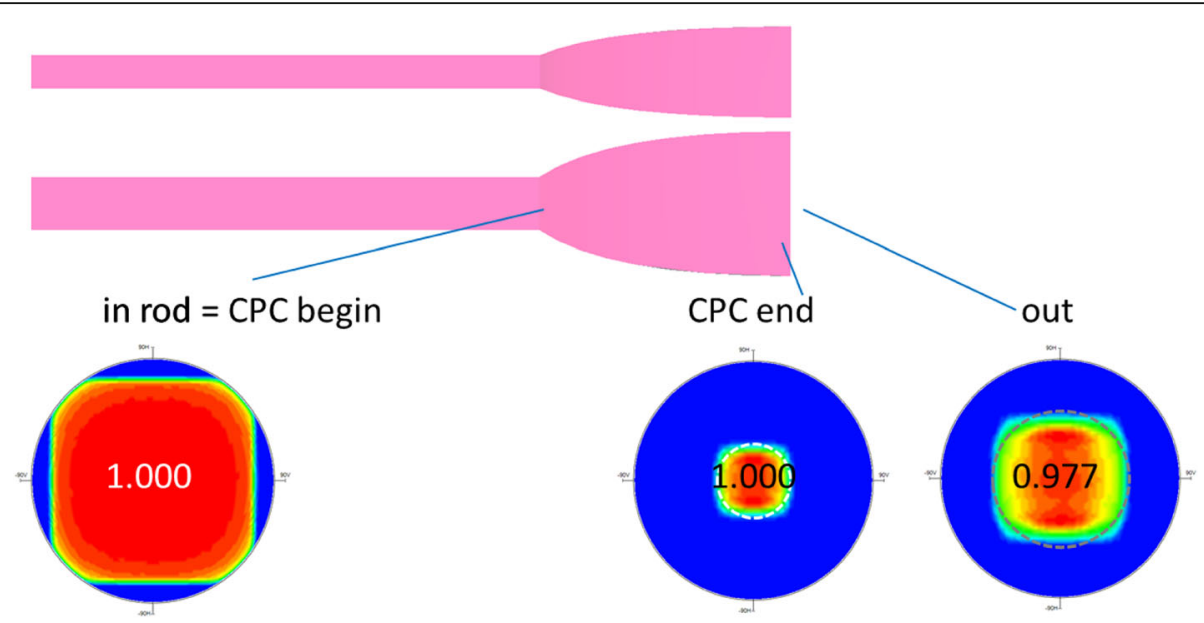

Fig. 7 Side views of a LuAG:Ce rod with a high-index ( $n=1.83$ ) CPC attached to it with simulated angular distributions of light at different stages. The numbers in the angular distributions show the fractions of light at the different stages 


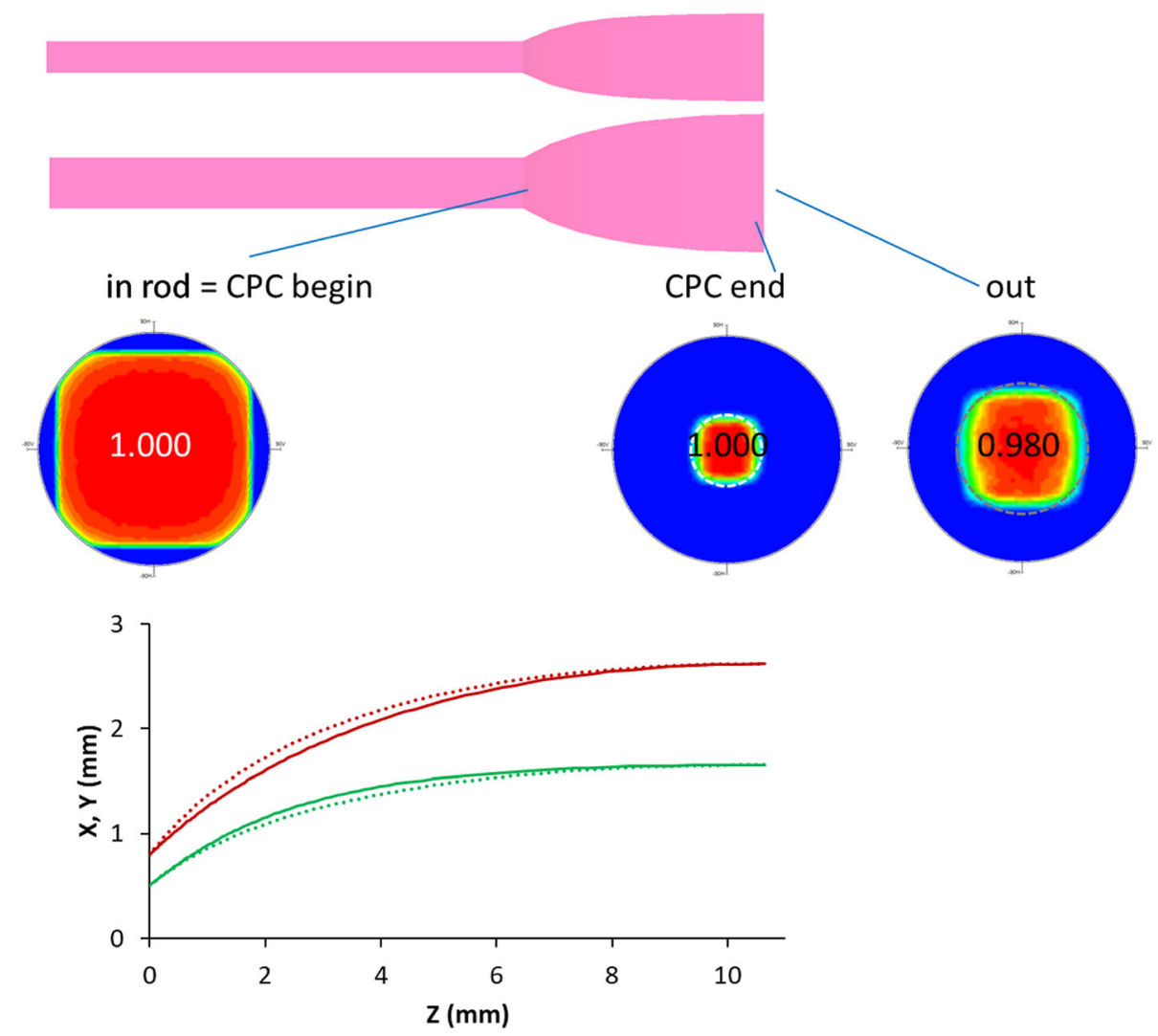

Fig. 8 Side views of a LUAG:Ce rod with a high-index $(n=1.83)$ concentrator with optimized Bézier shape attached to it with simulated angular distributions of light at different stages. The numbers in the angular distributions show the fractions of light at the different stages. At the bottom the Bézier shapes of the two sides are shown (solid lines) together with the CPC shapes (dotted) of Fig. 7

be coupled out. Due to scatter and reabsorption plus reemission, however, light can change direction. Part of it will escape from the sides, but a fraction 0.263 is found to stay in TIR, an appreciable part of which is at smaller angles than $56^{\circ}$. A fraction 0.175 is reflected again and 0.088 is extracted into the glass. This will continue and add up to a total fraction 0.827 of extracted light. We consider it as non-trivial that light outside the étendue of $16.5 \mathrm{~mm}^{2}$ sr can be forced back into a cone with half top angle $56^{\circ}$. According to Eq. (1), the law of étendue conservation can be broken if light conversion occurs. In this case, the small difference between the energies of reabsorbed and reemitted light is sufficient to reduce the étendue of the reflected light. This energy shift between light before and after recycling in the rod can be observed in the spectra shown in Fig. 5.

Next, we concentrate on the collimation efficiency of the CPC. There are two effects in a rectangular CPC that reduce the efficiency. In the first place, part of the light may leak from the CPC because it hits the surface at angles that do not yield TIR. Whereas a fraction 0.827 enters the $\mathrm{CPC}$, at the end a fraction 0.808 is left and a fraction 0.019 is lost by leakage. For the second effect, we look at the efficiency of the CPC for concentrating light in the intended étendue. At the right of Fig. 5, the angular distributions are shown at the end of the CPC in glass and outside the CPC in air. As can be seen, these distributions are more square than round. Circles are drawn indicating the aimed distribution cone with étendue $16.5 \mathrm{~mm}^{2}$ sr, i.e. with angles in glass up to $22^{\circ}$ and in air up to $34^{\circ}$. The corresponding fraction within this étendue is 0.741 (in glass and 0.734 in air).

One may ask whether it is possible to improve these numbers by adapting the shape of the concentrator. To this aim, the (stretched) parabolic shapes are replaced by Bézier curves of which the shape is optimized such that it gives a maximal light fraction at the end of the concentrator within the aimed distribution cone (with angles in glass up to $22^{\circ}$ ). In the optimization process, the dimensions at begin and end of the concentrator were kept fixed. The resulting concentrator shape and angular distributions are shown in Fig. 6. At the bottom of Fig. 6, the shapes are compared with those of the CPC of Fig. 5. The main difference is that the stretched CPC (with entrance width $1.2 \mathrm{~mm}$ ) is replaced by a shape with higher curvature. This has the effect that less light can escape 
from the concentrator and indeed the light leakage is found to be reduced to a fraction 0.007 (instead of 0.019) and the fraction of light at the end of the concentrator is 0.820 (instead of 0.808). The fraction of light within the intended étendue, however, only marginally increases to 0.749 (instead of 0.741). Apparently, a relatively higher amount of light ends up outside the intended étendue.

To couple all light from the rod end into the concentrator, the refractive indices of both should be equal, that is, $n=1.83$. However, since the étendue scales with the square of the refractive index of the concentrator, the dimensions of the cross sections of both rod and concentrator should be reduced by a factor 1.52/1.83 to ensure the same intended exit étendue $\left(16.5 \mathrm{~mm}^{2} \mathrm{sr}\right)$. In Fig. 7 , simulation results are shown for the case of a rod of with dimensions $64 \times 1.6 \times 1.0 \mathrm{~mm}^{3}$ and a concentrator with $n=1.83$ consisting of two two-dimensional CPCs. In this case, the square angular distribution at the end of the rod (and the begin of the concentrator) has an étendue of only $14 \mathrm{~mm}^{2}$ sr. For the CPC, an entrance angle $\theta=90^{\circ}$ is used to ensure that all light at skew angles is transmitted, aiming at an exit étendue of $16.5 \mathrm{~mm}^{2} \mathrm{sr}$. In this case, it is found that no leakage in the CPC occurs and the fraction of light at the CPC end is 1.000. As can be seen in Fig. 7, the resulting squarish angular distribution extends outside the cone (dashed line) with the intended étendue. Within this étendue, there is a fraction of 0.919 .

Also in this case, it is possible to optimize the shape by using Bézier curves. The result is shown in Fig. 8. In this case, mainly the shape of the side with entrance width $1.9 \mathrm{~mm}$ has altered. Apparently, this results in a rounder light distribution, of which a fraction 0.959 is in the desired étendue. It was found that the light leakage remains zero.

\section{Conclusions}

High lumen density sources (luminance $>2.10^{9} \mathrm{~cd} / \mathrm{m}^{2}$ ) based on luminescent concentration of converted LED light are promising for applications demanding high brightness. There are prospects to improve the performance even more, one of which is the optimization of the concentrator used as extraction optics. For this optimization, much insight can be obtained by raytracing simulations, especially if the angular and spectral light distributions at various stages in the system is considered.

For the extraction optics, a concentrator with high refractive index is preferred. From the simulations, it can be concluded that the efficiency can be improved by more than $20 \%$ if a high-index concentrator could be used. As a first prototype, we made a high-index $(n=1.8)$ CPC with entrance face $1.9 \times 1.2 \mathrm{~mm}^{2}$, attached to a LuAG:Ce rod. The measured conversion efficiency of blue to green light improved by $10 \%$, if compared to a low-index $(n=1.52)$ CPC. So, indeed there is potential for this technology.

Furthermore, it has been shown that the shape of the concentrator can be optimized. To this aim, the concentrator surfaces were parametrized as Bézier curves, of which the parameters are optimized. For a low-index $(n=1.52)$ concentrator, the light leakage can be reduced, but the gain in useful light is only marginal. For a highindex concentrator, the gain in useful light is somewhat more (a few percent). Such optimized concentrators can be manufactured by several techniques, e.g. glass moulding. The method of optimization can be more generally applied to maximize the flux of light in a desired spatial and/or angular region.

\section{Acknowledgements}

The authors would like to thank the following persons from Signify for useful input: Piet Antonis, Dominique Bruls, Christoph Hoelen, Henri Jagt, Jan Jansen, Yun Li, Patrick Van De Voorde, Peter Vanpoucke.

\section{Authors' contributions}

Both authors contributed to the simulations and analysis of the experiments. Both authors wrote the manuscript and approved the final manuscript.

\section{Funding}

Not applicable.

Availability of data and materials

The data supporting the conclusions of this article are included within the present article.

\section{Competing interests}

The authors declare that they have no competing interests.

Received: 13 March 2019 Accepted: 20 May 2019

Published online: 04 June 2019

\section{References}

1. de Boer, D.K.G., Bruls, D., Jagt, H.: High-brightness source based on luminescent concentration. Opt. Express. 24, A1069 (2016)

2. Hoelen, C., de Boer, D.K.G., Bruls, D., van der Eyden, J., Koole, R., Li, Y., Mirsadeghi, M., Vanbroekhoven, V., Van den Bergh, J.-J., Van de Voorde, P.: LED light engine concept with ultra-high scalable luminance. Proc. SPIE. 9768, 9768-9763 (2016)

3. de Boer, D.K.G., Bruls, D., Hoelen, C., Jagt, H.: High lumen density sources based on LED-pumped phosphor rods: opportunities for performance improvement. Proc. SPIE. 9896, 9896-9806 (2016)

4. de Boer, D.K.G., Bruls, D., Jagt, H., Hoelen, C.: LED-based projection source based on luminescent concentration. Proc. SPIE. 10378, 103780M (2017)

5. Hoelen, C., Antonis, P., de Boer, D., Koole, R., Kadijk, S., Li, Y., Vanbroekhoven, $\checkmark$., Van de Voorde, P.: Progress in extremely high brightness LED-based light sources. Proc. SPIE. 10378, 103780N (2017)

6. Barbet, A., Paul, A., Gallinelli, T., Balembois, F., Blanchot, J.-P., Forget, S., Chénais, S., Druon, F., Georges, P.: Light-emitting diode pumped luminescent concentrators: a new opportunity for low-cost solid-state lasers. Optica. 3, 465 (2016)

7. Sathian, J., Breeze, J.D., Richards, B., Alford, N.M., Oxborrow, M.: Solid-state source of intense yellow light based on a Ce: YAG luminescent concentrator. Opt. Express 25, 13714 (2017)

8. Goetzberger, A., Greubel, W.: Solar energy conversion with fluorescent collectors. Appl. Phys. 14, 123-139 (1977)

9. Debije, M.G., Verbunt, P.P.C.: Thirty years of luminescent solar concentrator research: solar energy for the built environment. Adv. Energy Mater. 2 12-35 (2012) 
10. de Boer, D.K.G., Broer, D.J., Debije, M.G., Keur, W., Meijerink, A., Ronda, C.R., Verbunt, P.P.C.: Progress in phosphors and filters for luminescent solar concentrators. Opt. Express. 20, A395 (2012)

11. McKenna, B., Evans, R.C.: Towards efficient spectral converters through materials design for luminescent solar devices. Adv. Mater. 29, 1 (2017)

12. Yablonovitch, E.: Thermodynamics of the fluorescent planar concentrator. J. Opt. Soc. Am. 70, 1362-1363 (1980)

13. Roelandt, S., Meuret, Y., de Boer, D.K.G., Bruls, D., Van De Voorde, P., Thienpont, H.: In- and Outcoupling of Light from a Luminescent Rod Using a Compound Parabolic Concentrator (CPC). Optical Eng. 54, 055101 (2015)

14. Sellami, N., Mallick, T.P.: Optical efficiency study of PV crossed compound parabolic concentrator. Appl. Energy. 102, 868 (2013)

15. Davenport, T.L.R., Hough, T.A., Cassarly, W.J.: Optimization for efficient angleto-area conversion in illumination systems. Proc. SPIE. 5524, 93 (2004)

\section{Publisher's Note}

Springer Nature remains neutral with regard to jurisdictional claims in published maps and institutional affiliations.

\section{Submit your manuscript to a SpringerOpen ${ }^{\odot}$ journal and benefit from:}

- Convenient online submission

- Rigorous peer review

- Open access: articles freely available online

- High visibility within the field

- Retaining the copyright to your article

Submit your next manuscript at $\boldsymbol{\nabla}$ springeropen.com 